\title{
Maximum matchings in regular graphs of high girth
}

\author{
Abraham D. Flaxman \\ Microsoft Research \\ Redmond, Washington, USA \\ abie@microsoft.com
}

\author{
Shlomo Hoory \\ IBM Research Laboratory \\ Haifa, Israel \\ shlomoh@il.ibm.com
}

\author{
Submitted: April 22, 2006; Accepted: Dec 10, 2006; Published: Jan 3, 2007 \\ Mathematics Subject Classification: 05C70
}

\begin{abstract}
Let $G=(V, E)$ be any $d$-regular graph with girth $g$ on $n$ vertices, for $d \geq 3$. This note shows that $G$ has a maximum matching which includes all but an exponentially small fraction of the vertices, $O\left((d-1)^{-g / 2}\right)$. Specifically, in a maximum matching of $G$, the number of unmatched vertices is at most $n / n_{0}(d, g)$, where $n_{0}(d, g)$ is the number of vertices in a ball of radius $\lfloor(g-1) / 2\rfloor$ around a vertex, for odd values of $g$, and around an edge, for even values of $g$. This result is tight if $n<2 n_{0}(d, g)$.
\end{abstract}

\section{Introduction}

For a graph $G$, a matching (or one-factor) of $G$ is a subgraph of $G$ in which each vertex has degree at most one. A basic question in combinatorial optimization is, "What is the size of a maximum matching in a given graph?" This has been explored extensively as an algorithmic question, and a number of efficient procedures are known which find a maximum matching of a given graph. This note considers the question from a different angle. What general graph properties imply that a graph must contain a large matching? This note shows that in regular graphs, high girth is such a property.

The notation used in this note is collected in this paragraph. For the graph $G=(V, E)$ and $u, v \in V$, write $u \sim v$ if and only if $\{u, v\} \in E$, and abbreviate $\{u, v\}$ with $u v$ when it is clear from context that this refers to an edge. For $S \subseteq V$, write $G[S]$ to denote the graph $G$ induced by the vertices $S$, and write $E[S]$ to denote the edges in $G[S]$. The girth of a graph $G$ is the length of the shortest cycle in $G$. When dealing with a different graph, say $H$, write $V(H)$ to denote the vertex set of $H$, and $E(H)$ to denote the edge set. Finally, say that $G$ is a $(d, g)$-graph if it is a $d$-regular graph of girth at least $g$. 
Theorem 1 Let $G$ be a $(d, g)$-graph on $n$ vertices and $d \geq 2$. Then $G$ contains a matching with at most $n / n_{0}(d, g)$ unmatched vertices, where

$$
\begin{aligned}
n_{0}(d, 2 r+1) & =1+d \sum_{i=0}^{r-1}(d-1)^{i}, \\
n_{0}(d, 2 r) & =2 \sum_{i=0}^{r-1}(d-1)^{i} .
\end{aligned}
$$

Theorem 1 shows that for $d$-regular graphs with fixed $d \geq 3$, the fraction of unmatched vertices in a maximum matching is exponentially small in the girth of the graph. For 2regular graphs (which is to say, collections of disjoint cycles) the fraction of unmatched vertices tends to 0 like $1 / \mathrm{g}$.

The bound in the theorem is tight in some cases. To argue this requires some background information. Let $n(d, g)$ be the least number of vertices in a $(d, g)$-graph. The simplest lower bound on $n(d, g)$ is the Moore bound, which states that $n(d, g) \geq n_{0}(d, g)$. It can be derived by a counting argument based on the fact that a ball of radius $\left\lfloor\frac{g-1}{2}\right\rfloor$ around a vertex is a tree (or a ball around an edge, depending on the parity of $g$ ) [3, Proposition 23.1, p. 180]. Although significant effort has been invested in improving this lower bound, the best known bound is still $n_{0}(d, g)+c$ for some small additive constant (see [3, Theorem 23.6, p. 185], and the survey [9]). On the other hand, the best upper bound on $n(d, g)$ is on the order of $n_{0}(d, g)^{3 / 2}$, and is achieved by several constructions $[7,8,6]$. Establishing better asymptotic bounds on $n(d, g)$ is a well known open question.

That Theorem 1 is tight for certain values of $(d, g)$ can be seen as follows. The theorem implies that a $(d, g)$-graph $G$ of size $n<2 n_{0}(d, g)$ has at most one unmatched vertex. Therefore, since the number of unmatched vertices must have the same parity as $n$, this result is tight. In particular, if $n$ is even, then the graph has a perfect matching. As mentioned previously, it is not known if there are pairs $(d, g)$ such that $n(d, g)<2 n_{0}(d, g)$ for $d \geq 3$ and large $g$. However, there are abundant examples of such $(d, g)$-graphs for small values of $g$. It is worth mentioning that a result of [1] implies that under the same conditions the graph $G$ is an expander. Namely, for any integers $d \geq 3$ and $g$ and a graph $G$ satisfying $n(d, g)<2 n_{0}(d, g)$, the second largest eigenvalue of the adjacency matrix $\lambda(G)$ is smaller than $d-\epsilon$ for some $\epsilon=\epsilon(d, g)>0$.

The proof of Theorem 1 is a simple combination of the correct ingredients. One ingredient is the characterization of the matching polytope due to Edmonds [5]. (See also the recent survey by Cunningham for several different proofs and a historical discussion [4].)

Theorem 2 (Edmonds, 1965) The set of incidence vectors of the matchings of $G$ have a convex hull which is equal to the polytope containing all $x \in \mathbb{R}^{E}$ which satisfy the following 
three conditions:

$$
\begin{array}{rlrl}
\sum_{w: v \sim w} x_{v w} & \leq 1, & & \text { for all } v \in V ; \\
\sum_{e \in E[S]} x_{e} \leq \frac{|S|-1}{2}, & & \text { for all } S \subseteq V \text { with }|S| \text { odd } \\
x_{e} \geq 0, & \text { for all } e \in E .
\end{array}
$$

Another ingredient is the generalization of the Moore bound to irregular graphs, due to Alon, Hoory, and Linial [2]. As stated before, the original Moore bound applies to regular graphs only and states that $n(d, g) \geq n_{0}(d, g)$.

Theorem 3 (Alon, Hoory, and Linial, 2001) The number of vertices $n$ in a graph of girth $g$ and average degree $d \geq 2$ satisfies

$$
n \geq n_{0}(d, g) .
$$

With these theorems in hand, it is a simple matter to verify that a point $\bar{z} \in \mathbb{R}^{E}$, which will be specified explicitly in the next section, lies within the matching polytope. Since the extreme points of the matching polytope are integral, this shows that there is some integral solution to the LP which corresponds to a matching with at least as many edges as the weight of $\bar{z}$.

\section{Proof of Theorem 1}

For $d=2$, the theorem states that the portion of unmatched vertices is at most $1 / n_{0}(d, g)=$ $1 / g$. The result in this case follows by observing that $G$ is a collection of cycles of length at least $g$, and each cycle has at most one unmatched vertex in a maximum matching. Henceforce, consider the degree $d$ to be at least 3 .

Consider the point $\bar{z}=(z, \ldots, z) \in \mathbb{R}^{E}$. Then $\bar{z}$ is in the matching polytope if $0 \leq z \leq 1 / d$ and for every vertex set $S$ of odd size

$$
z \cdot|E[S]| \leq \frac{|S|-1}{2}
$$

Let $d_{S}=2|E[S]| /|S|$ denote the average degree of the subgraph $G[S]$. If $d_{S}<2$ then $|E[S]| \leq|S|-1$ which yields the required inequality. So assume that $d_{S} \geq 2$ and rewrite (4) as:

$$
z \leq \frac{|S|-1}{d_{S} \cdot|S|}
$$

The right hand side of the inequality is increasing as a function of $|S|$, so, by Theorem 3 , for the inequality to hold, it is sufficient to take $z$ to be the minimum of $f(\delta)$ where $2 \leq \delta \leq d$ and

$$
f(\delta)=\frac{n_{0}(\delta, g)-1}{\delta n_{0}(\delta, g)}
$$


It is shown below that $f(\delta)$ is monotone decreasing for $\delta \geq 2$, so that the minimum is attained by $\delta=d$. Therefore there is a matching of size at least the weight of $\bar{z}$, which is

$$
f(d) \cdot \frac{n d}{2}=\left(1-\frac{1}{n_{0}(d, g)}\right) \cdot \frac{n}{2},
$$

as claimed.

It remains to show that $f(\delta)$ is monotone decreasing for $\delta \geq 2$, i.e.

$$
f^{\prime}(\delta)=\frac{d}{d \delta}\left(\frac{1}{\delta}-\frac{1}{\delta n_{0}(\delta, g)}\right) \leq 0
$$

This is equivalent to

$$
n_{0}(\delta, g)+\delta n_{0}^{\prime}(\delta, g) \leq n_{0}(\delta, g)^{2} .
$$

Since $n_{0}(\delta, g)$ is a polynomial of degree $r=\lfloor(g-1) / 2\rfloor$ in $\delta-1$ with non-negative coefficients, then $(\delta-1) n_{0}^{\prime}(\delta, g) \leq r n_{0}(\delta, g)$ for all $\delta \geq 2$. Therefore

$$
n_{0}(\delta, g)+\delta n_{0}^{\prime}(\delta, g) \leq n_{0}(\delta, g)+2(\delta-1) n_{0}^{\prime}(\delta, g) \leq(2 r+1) n_{0}(\delta, g) \leq g n_{0}(\delta, g) \leq n_{0}(\delta, g)^{2},
$$

which verifies that inequality (5) holds. This proves the monotonicity of $f(\delta)$ in the required range, and completes the proof of the theorem.

\section{References}

[1] A. Alon, S. Hoory, N. Linial, A Continuous Analogue of the Girth Problem, Journal of Combinatorial Theory Series B 84(2), p.340-363, 2002.

[2] N. Alon, S. Hoory, N. Linial, The Moore bound for irregular graphs, Graph and Combinatorics 18(1), p.53-57, 2002.

[3] N. Biggs, Algebraic Graph Theory, Cambridge University Press, Cambridge, 2nd Ed. (1993).

[4] W. H. Cunningham, Matchings, matroids, and extensions, Math. Program., Ser. B, 91 (2002) 515-542.

[5] J. Edmonds, Maximum matching and a polyhedron with 0, 1 vertices, J. Res. Nat. Bur. Standards Sect. B 69, 125-130.

[6] F. Lazebnik, V. A. Ustimenko, A. Woldar, A new series of dense graphs of high girth, Bull. Amer. Math. Soc. (New Series), 32 issue 1 (1995), 73-79.

[7] A. Lubotzky, R. Phillips, P. Sarnak, Ramanujan graphs, Combinatorica 8 issue 3 (1988). $261-277$.

[8] G. A. Margulis, Explicit group-theoretic constructions of combinatorial schemes and their applications in the construction of expanders and concentrators, Probl. of Inform. Trans., 24 issue 1 (1988),39-46.

[9] P. K. Wong, Cages-a survey, J. Graph Theory 6 (1982), 1-22. 